\title{
Front Matter: Volume 7913
}

, "Front Matter: Volume 7913," Proc. SPIE 7913, Laser Resonators and Beam Control XIII, 791301 (5 April 2011); doi: 10.1117/12.890182

SPIE. Event: SPIE LASE, 2011, San Francisco, California, United States 


\title{
PROCEEDINGS OF SPIE
}

\section{Laser Resonators and Beam Control XIII}

\author{
Alexis V. Kudryashov \\ Alan H. Paxton \\ Vladimir S. Ilchenko \\ Lutz Aschke \\ Editors
}

23-25 January 2011

San Francisco, California, United States

Sponsored and Published by

SPIE 
The papers included in this volume were part of the technical conference cited on the cover and title page. Papers were selected and subject to review by the editors and conference program committee. Some conference presentations may not be available for publication. The papers published in these proceedings reflect the work and thoughts of the authors and are published herein as submitted. The publisher is not responsible for the validity of the information or for any outcomes resulting from reliance thereon.

Please use the following format to cite material from this book:

Author(s), "Title of Paper," in Laser Resonators and Beam Control XIII, edited by

Alexis V. Kudryashov, Alan H. Paxton, Vladimir S. Ilchenko, Lutz Aschke, Proceedings of SPIE Vol. 7913 (SPIE, Bellingham, WA, 2011) Article CID Number.

ISSN 0277-786X

ISBN 9780819484505

Published by

SPIE

P.O. Box 10, Bellingham, Washington 98227-0010 USA

Telephone +1 3606763290 (Pacific Time) · Fax +1 3606471445

SPIE.org

Copyright (C) 2011, Society of Photo-Optical Instrumentation Engineers

Copying of material in this book for internal or personal use, or for the internal or personal use of specific clients, beyond the fair use provisions granted by the U.S. Copyright Law is authorized by SPIE subject to payment of copying fees. The Transactional Reporting Service base fee for this volume is $\$ 18.00$ per article (or portion thereof), which should be paid directly to the Copyright Clearance Center (CCC), 222 Rosewood Drive, Danvers, MA 01923. Payment may also be made electronically through CCC Online at copyright.com. Other copying for republication, resale, advertising or promotion, or any form of systematic or multiple reproduction of any material in this book is prohibited except with permission in writing from the publisher. The CCC fee code is 0277-786X/11/ \$18.00.

Printed in the United States of America.

Publication of record for individual papers is online in the SPIE Digital Library.

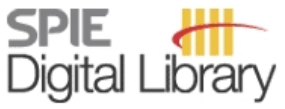

SPIEDigitalLibrary.org

Paper Numbering: Proceedings of SPIE follow an e-First publication model, with papers published first online and then in print and on CD-ROM. Papers are published as they are submitted and meet publication criteria. A unique, consistent, permanent citation identifier (CID) number is assigned to each article at the time of the first publication. Utilization of CIDs allows articles to be fully citable as soon as they are published online, and connects the same identifier to all online, print, and electronic versions of the publication. SPIE uses a six-digit CID article numbering system in which:

- The first four digits correspond to the SPIE volume number.

- The last two digits indicate publication order within the volume using a Base 36 numbering system employing both numerals and letters. These two-number sets start with 00, 01, 02, 03, 04 , 05, 06, 07, 08, 09, OA, OB ... 0Z, followed by 10-1Z, 20-2Z, etc.

The CID number appears on each page of the manuscript. The complete citation is used on the first page, and an abbreviated version on subsequent pages. Numbers in the index correspond to the last two digits of the six-digit CID number. 


\section{Contents}

ix Conference Committee

xi Microfabrication by optical tweezers [792102]

R. Ghadiri, T. Weigel, C. Esen, A. Ostendorf, Ruhr-Univ. Bochum (Germany)

\section{SESSION1 LASER RESONATOR AND GAIN MEDIUM SIMULATION}

791302 Unstable ring resonator with bidirectional propagation through the gain medium: analysis [7913-01]

A. H. Paxton, Air Force Research Lab. (United States)

791303 Analysis of frequency dependent pump light absorption [7913-02]

M. Wohlmuth, C. Pflaum, Friedrich-Alexander-Univ. Erlangen-Nürnberg (Germany)

791304 Constructing petal modes from the coherent superposition of Laguerre-Gaussian modes [7913-03]

D. Naidoo, A. Forbes, Council for Scientific and Industrial Research (South Africa) and Univ. of KwaZulu-Natal (South Africa); K. Aït-Ameur, Ecole Nationale Supérieure d'Ingenieurs de Caen et Ctr. de Recherche (France); M. Brunel, Univ. de Rouen (France)

791305 Laser sustained plasma ball lensing effect controlled by means of coaxial gas flow [7913-04]

V. P. Zimakov, A. Y. Kedrov, V. A. Kuznetsov, A. N. Shemyakin, N. G. Solovyov, M. Y. Yakimov, A.Ishlinsky Institute for Problems in Mechanics (Russian Federation)

\section{SESSION 2 LASER DIAGNOSTICS AND COMPONENTS}

791306 Real time laser beam analysis system for high power lasers [7913-05]

M. Scaggs, Neoteric Concepts, LLC (United States); G. Haas, Haas Laser Technologies, Inc. (United States)

791307 Enhanced high-speed coherent diffraction imaging [7913-06]

J. Potier, S. Fricker, PhaseView (France); M. Idir, Brookhaven National Lab. (United States)

791309 Shack-Hartmann wavefront sensor and its problems (Invited Paper) [7913-08]

A. Kudryashov, V. Zavalova, A. Rukosuev, A. Alexandrov, J. Sheldakova, V. Samarkin, Moscow State Open Univ. (Russian Federation)

\section{SESSION $3 \quad$ LASER BEAM SHAPING}

7913 OB Efficient diffractive optical elements from glass with continuous surface profiles [7913-10]

Y. V. Miklyaev, A. Krasnaberski, M. Ivanenko, A. Mikhailov, W. Imgrunt, L. Aschke,

V. N. Lissotschenko, LIMO Lissotschenko Mikrooptik GmbH (Germany) 
7913 OC Thermal lensing compensation optics for high power lasers [7913-11]

M. Scaggs, Neoteric Concepts, LLC (United States); G. Haas, Haas Laser Technologies, Inc. (United States)

7913 OD Adaptive extracavity beam shaping for application in nanosecond laser micromachining [7913-12]

R. J. Beck, J. P. Parry, J. D. Shephard, D. P. Hand, Heriot-Watt Univ. (United Kingdom)

7913 OE Multistage phased electro-optical planar arrays for the manipulation of high power laser beams [7913-13]

M. Ivanenko, A. Krasnaberski, A. Mikhailov, Y. Miklyaev, L. Aschke, V. Lissotschenko, LIMO Lissotschenko Mikrooptik GmbH (Germany)

7913 OF Laser beam alignment and profilometry using diagnostic fluorescent safety mirrors [7913-14] T. E. Lizotte, Hitachi Via Mechanics (USA), Inc. (United States)

\section{SESSION 4 RESONATORS AND ADAPTIVE OPTICS}

7913 OG Negative-branch unstable resonator in off-axis configuration for rectangular cross-sections [7913-15]

C. Pargmann, T. Hall, F. Duschek, K. M. Grünewald, J. Handke, Deutsches Zentrum für Luft- und Raumfahrt e.V. (Germany)

$7913 \mathrm{OH} \quad$ Adaptive optics on Petawatt lasers: current performance of the Texas Petawatt Laser (Invited Paper) [7913-17]

M. Martinez, E. Gaul, T. Borger, F. Aymond, D. Hammond, M. Ringuette, R. Escamilla,

T. Ditmire, The Univ. of Texas at Austin (United States); J. Caird, A. Erlandson, I. Iovanovic,

C. Ebbers, B. Molander, Lawrence Livermore National Lab. (United States)

7913 Ol Laser beam formation by adaptive optics [7913-18]

J. Sheldakova, V. Samarkin, A. Kudryashov, A. Rukosuev, Active Optics NightN Ltd.

(Russian Federation) and Moscow State Open Univ. (Russian Federation)

$79130 \mathrm{~J} \quad$ Applying of refractive beam shapers of circular symmetry to generate non-circular shapes of homogenized laser beams [7913-20]

A. Laskin, Molecular Technology GmbH (Germany); V. Laskin, AdlOptica GmbH (Germany)

\section{SESSION 5 MICRORESONATORS: NOVEL MORPHOLOGIES AND DEVICE APPLICATIONS I}

$7913 \mathrm{OL} \quad$ Filters and electro-optic modulators on fiber end-faces [7913-42]

S. Meister, D. Schweda, M. Dziedzina, R. Juhre, A. Al-Saadi, B. A. Franke, Technische Univ. Berlin (Germany); B. Grimm, S. K. Schrader, Technische Fachhochschule Wildau (Germany); S. J. Benight, D. H. Bale, I. Kosilkin, L. R. Dalton, Univ. of Washington (United States);

H. J. Eichler, Technische Univ. Berlin (Germany)

7913 ON Ultra-high Q whispering-gallery-mode bottle microresonators: properties and applications (Invited Paper) [7913-22]

D. O'Shea, C. Junge, Technische Univ. Wien (Austria); S. Nickel, M. Pöllinger, Johannes Gutenberg Univ. Mainz (Germany); A. Rauschenbeutel, Technische Univ. Wien (Austria) 
791300 A hybrid quantum photonic interface for solid state qubits (Invited Paper) [7913-23] D. Englund, L. Li, J. Hodges, Columbia Univ. (United States); B. Shields, Harvard Univ. (United States); K. Rivoire, Stanford Univ. (United States); F. Hatami, Humboldt Univ. (Germany); J. Vuckovic, Stanford Univ. (United States); H. Park, M. Lukin, Harvard Univ. (United States)

\section{SESSION $6 \quad$ MICRORESONATORS: FREQUENCY COMBS}

$79130 Q \quad$ Spectrum engineering in whispering gallery mode resonators [7913-25]

A. B. Matsko, A. A. Savchenkov, W. Liang, V. S. Ilchenko, D. Seidel, L. Maleki, OEwaves, Inc. (United States)

7913 OT Challenges in octave-spanning and short free-spectral-range optical frequency comb generation using monolithic whispering gallery mode resonators (Invited Paper) [7913-28]

Y. K. Chembo, FEMTO-ST Institute, CNRS (France); N. Yu, Jet Propulsion Lab. (United States)

\section{SESSION 7 MICRORESONATORS: LASERS AND PHOTONICS DEVICES}

7913 OV Multiple-port directional emission whispering-gallery mode microlasers (Invited Paper) [7913-30]

Y.-Z. Huang, Y.-D. Yang, J.-D. Lin, K.-J. Che, S.-J. Wang, J.-L. Xiao, Y. Du, Institute of Semiconductors (China)

7913 OY Lasing in dye-doped high-Q conical polymeric microcavities [7913-33] T. Grossmann, S. Schleede, M. Hauser, Karlsruher Institut für Technologie (Germany); M. B. Christiansen, Technical Univ. of Denmark (Denmark); C. Vannahme, C. Eschenbaum, S. Klinkhammer, T. Beck, J. Fuchs, G. U. Nienhaus, U. Lemmer, Karlsruher Institut für Technologie (Germany); A. Kristensen, Technical Univ. of Denmark (Denmark); T. Mappes, H. Kalt, Karlsruher Institut für Technologie (Germany)

791310 Gain-guided leaky-waveguide laser amplifiers: analytical methods and recent progress on experiments [7913-35]

T.-H. Her, L. W. Casperson, C. Wang, L. Zhao, The Univ. of North Carolina at Charlotte (United States)

\section{SESSION 8 MICRORESONATORS: SENSORS}

791312 A self-reference sensing technique for ultra-sensitive chemical and biological detection using whispering gallery microresonators (Invited Paper) [7913-37]

L. Yang, J. Zhu, S. K. Ozdemir, L. He, W. Kim, D.-R. Chen, Washington Univ. in St. Louis (United States)

791313 Microring and microdisk resonator-based devices for on-chip optical interconnects, particle manipulation, and biosensing (Invited Paper) [7913-38]

A. W. Poon, S. Feng, H. Cai, T. Lei, H. Chen, X. Luo, Hong Kong Univ. of Science and Technology (Hong Kong, China) 
791314 Characterization of high index microsphere resonators in fiber-integrated microfluidic platforms [7913-39]

O. Svitelskiy, D. Sun, A. Darafsheh, The Univ. of North Carolina at Charlotte (United States); M. Sumetsky, OFS Labs. (United States); A. Lupu, M. Tchernycheva, Institut d'Electronique Fondamentale, CNRS, Univ. Paris-Sud 11 (France); V. N. Astratov, The Univ. of North Carolina at Charlotte (United States)

\section{SESSION 9 MICRORESONATORS: NOVEL MORPHOLOGIES AND DEVICE APPLICATIONS II}

791317 Polarization-purity spectra of a tapered-fiber-coupled microsphere cavity system at cryogenic temperatures [7913-43] M. Fujiwara, A. Tanaka, K. Toubaru, H.-Q. Zhao, H. Takashima, S. Takeuchi, Hokkaido Univ. (Japan) and Osaka Univ. (Japan)

791319 Heterogeneously integrated microdisk lasers for optical interconnects and optical logic (Invited Paper) [7913-51]

P. Méchet, L. Liu, R. Kumar, K. Huybrechts, T. Spuesens, G. Roelkens, Univ. Gent (Belgium); E.-J. Geluk, T. de Vries, Technische Univ. Eindhoven (Netherlands); P. Regreny, INL-UMR5270, CNRS, Ecole Centrale de Lyon (France); D. Van Thourhout, R. Baets, G. Morthier, Univ. Gent (Belgium)

$79131 \mathrm{~A} \quad$ Focusing capability of integrated chains of microspheres in the limit of geometrical optics [7913-45]

A. Darafsheh, K. W. Allen, The Univ. of North Carolina at Charlotte (United States); A. Fardad, PhotonTech LLC (United States); N. M. Fried, The Univ. of North Carolina at Charlotte (United States); A. N. Antoszyk, Charlotte Eye Ear Nose and Throat Associates (United States); H. S. Ying, Johns Hopkins Univ. (United States); V. N. Astratov, The Univ. of North Carolina at Charlotte (United States)

7913 1B Perturbation of whispering gallery modes of magnetorheological polydimethylsiloxane spheres by external magnetic field [7913-46]

T. Ioppolo, M. V. Ötügen, Southern Methodist Univ. (United States)

POSTER SESSION

7913 1C Hyper coherent linewidth control for a 30 $\mathrm{mW}$ 405nm visible laser diode by delayed self-heterodyne beat [7913-47]

M. Matsumura, T. Kuromori, W. Sasaki, Doshisha Univ. (Japan)

$79131 \mathrm{E} \quad$ ABOUT the phenomenon produced by the successive jumps of the peripheric electrons, at the absorbtion of the intense photon beam by the metal [7913-49]

C. I. Isarie, C. Oprean, I. Marginean, T. Nemes, I. V. Isarie, C. Bokor, S. Itu, Lucian Blaga Univ. (Romania)

7913 IF Intra-cavity decomposition of a dual-directional laser beam [7913-50]

D. Naidoo, A. Forbes, Council for Scientific and Industrial Research (South Africa) and Univ. of KwaZulu-Natal (South Africa); K. Aït-Ameur, M. Fromager, Ecole Nationale Supérieure d'Ingenieurs de Caen et Ctr. de Recherche (France) 


\section{LATE PAPER SESSION}

$79131 \mathrm{G}$ Compact tunable kHz-linewidth semiconductor laser stabilized with a whispering-gallery mode microresonator [7913-100]

V. S. Ilchenko, E. Dale, W. Liang, J. Byrd, D. Eliyahu, A. A. Savchenkov, A. B. Matsko, D. Seidel, L. Maleki, OEwaves, Inc. (United States)

Author Index 
Downloaded From: https://www.spiedigitallibrary.org/conference-proceedings-of-spie on 26 Apr 2023

Terms of Use: https://www.spiedigitallibrary.org/terms-of-use 


\title{
Conference Committee
}

\author{
Symposium Chairs
}

Friedhelm Dorsch, TRUMPF GmbH \& Company KG (Germany)

Alberto Piqué, U.S. Naval Research Laboratory (United States)

Symposium Cochairs

Donald J. Harter, IMRA America, Inc. (United States)

Peter R. Herman, University of Toronto (Canada)

Program Track Chair

Gregory J. Quarles, BE Meyers \& Company Inc. (United States)

Conference Chairs

Alexis V. Kudryashov, Moscow State Open University

(Russian Federation)

Alan H. Paxton, Air Force Research Laboratory (United States)

Vladimir S. Ilchenko, OEwaves, Inc. (United States)

Conference Cochair

Lutz Aschke, LIMO Lissotschenko Mikrooptik GmbH (Germany)

Program Committee

Jean-Claude Marcel Diels, The University of New Mexico

(United States)

Hans-Joachim Eichler, Technische Universität Berlin (Germany)

Andrew Forbes, Council for Scientific and Industrial Research

(South Africa)

Pierre Galarneau, INO (Canada)

Thomas Graf, Universität Stuttgart (Germany)

James R. Leger, University of Minnesota, Twin Cities (United States)

Andrey B. Matsko, OEwaves, Inc. (United States)

Shayan Mookherjea, University of California, San Diego (United States)

Steve A. Pappert, Defense Advanced Research Projects Agency

(United States)

Andrew W. Poon, Hong Kong University of Science and Technology

(Hong Kong, China)

Michelle L. Povinelli, The University of Southern California

(United States)

Michael J. Scaggs, Haas Laser Technologies, Inc. (United States) 
Session Chairs

1 Laser Resonator and Gain Medium Simulation

Michael J. Scaggs, Haas Laser Technologies, Inc. (United States)

2 Laser Diagnostics and Components

Alexis V. Kudryashov, Moscow State Open University

(Russian Federation)

3 Laser Beam Shaping

Lutz Aschke, LIMO Lissotschenko Mikrooptik GmbH (Germany)

$4 \quad$ Resonators and Adaptive Optics

Andrew Forbes, Council for Scientific and Industrial Research

(South Africa)

5 Microresonators: Novel Morphologies and Device Applications I

Vladimir S. Ilchenko, OEWaves, Inc. (United States)

6 Microresonators: Frequency Combs

Shayan Mookherjea, University of California, San Diego (United States)

$7 \quad$ Microresonators: Lasers and Photonics Devices

Andrey B. Matsko, OEWaves, Inc. (United States)

8 Microresonators: Sensors

Michelle L. Povinelli, The University of Southern California (United States)

9 Microresonators: Novel Morphologies and Device Applications II Shayan Mookherjea, University of California, San Diego (United States) 